\title{
The Effect of Chemotherapy on Serum Carnitine Levels and Fatigue in Chemotherapy Naive Medical Oncology Patients: A Pilot Study. Carnitine, Chemotherapy and Fatigue
}

Wolfgang M Marx ${ }^{1^{*}}$, LaisaTeleni' ${ }^{2}$, Maree Ferguson ${ }^{2}$, Euan Walpole ${ }^{3}$ and Elizabeth A Isenring ${ }^{1,2,4}$

${ }^{1}$ Centre for Dietetics Research, School of Human Movement Studies, University of Queensland, Brisbane, Queensland, Australia

${ }^{2}$ Department of Nutrition and Dietetics, Princess Alexandra Hospital, Metro South Hospital and Health Services, Brisbane, Queensland, Australia

${ }^{3}$ Cancer Services, Princess Alexandra Hospital, Metro South Hospital and Health Services, Brisbane, Queensland, Australia

${ }^{4}$ Wolf Marx, be amended to also include the fourth affiliation, Health Sciences \& Medicine, Bond University, Gold Coast, Queensland, Australia

\begin{abstract}
Background: Fatigue, a highly prevalent side-effect of anticancer therapy, poses a significant burden on patient quality of life. Carnitine deficiency has been implicated in the development of fatigue during chemotherapy.

Methods: This pilot study aimed to evaluate the relationship between chemotherapy-related fatigue and serum carnitine (total, free- and acyl: free carnitine ratio) in 35 chemotherapy-naïve oncology patients at baseline, 6 and 12 weeks.

Results: Carnitine deficiency, based on acyl: free carnitine ratio, was detected in three patients at baseline but this did not persist to the next time point. Carnitine deficiency was not detected in any other participant throughout this study and levels of carnitine were not correlated with fatigue during the study period.
\end{abstract}

Conclusion: A relationship between carnitine status and fatigue was not found at the time points measured Further research is required to confirm these results in a larger medical oncology population.

Keywords: Carnitine; Chemotherapy; Fatigue; Cancer; Clinical

\section{Introduction}

Fatigue is the most common side-effect reported by oncology patients undergoing cytotoxic treatment [1]. L-Carnitine is primarily involved in the regulation of acyl-carnitines and their subsequent transportation across the inner mitochondrial membrane for beta oxidation $[2,3]$. It has been suggested that carnitine deficiencies may contribute to fatigue associated with chemotherapy.

Multiple studies have evaluated carnitine status in oncology patients undergoing chemotherapy using serum and/or urinary markers of carnitine [4-11]. While not all studies found that chemotherapy resulted in carnitine deficiency, all studies found carnitine metabolism to be significantly impaired during the study period. In addition, chemotherapy regimens including doxorubicin, Platinum-based therapies (e.g. cisplatin), and ifosfamide have been shown to disrupt regular carnitine metabolism. Several potential mechanisms have been proposed including an increased rate of renal excretion $[5,8]$ a reduction in cellular uptake [12] and an increase in the metabolic demand for carnitine [13] therefore, prolonged exposure to these regimens may result in carnitine deficiencies [7]. In addition, most studies to date have only measured the acute changes in carnitine status during chemotherapy rather than over the course of multiple cycles which is typical of most chemotherapy regimens.

The aim of this study was to determine the prevalence of carnitine deficiency in patients undergoing chemotherapy over an extended period of time and to evaluate the relationship between chemotherapyrelated fatigue and serum carnitine levels. We hypothesized that carnitine levels would decline during the course of the study and that carnitine status is inversely correlated to patient fatigue.

\section{Methods}

This was a prospective cohort pilot study conducted at a metropolitan, tertiary teaching hospital. Data were collected at baseline (commencement of chemotherapy) \pm 1 week, $6 \pm 1$ week, and $12 \pm 1$ week. Approval by the Metro South Health Service District and University of Queensland Human Research Ethics Committees was granted and written informed consent was obtained from all participants before any study procedure was commenced.

\section{Eligibility}

Inclusion criteria: 1) chemotherapy-naïve; 2) commencing chemotherapy within 2 weeks of study entry; 3) histologicallyconfirmed neoplasm 4$) \geq 18$ years of age.

\section{Carnitine levels}

Blood samples were taken \pm 1 week of each time point, at the same time as routine blood tests. Serum carnitine measures included total carnitine, free carnitine, acyl: free carnitine ratio ((total-free carnitine)/ free carnitine), and the prevalence of carnitine deficiency as defined by free carnitine ( $<35 \mu \mathrm{mol} / \mathrm{L}$ for males and $<25 \mu \mathrm{mol} / \mathrm{L}$ for females) and acyl: free carnitine ratio $(>0.40)$ [14]. Carnitine was analysed using butyl ester derivatives and electro spray tandem mass spectrometry [15].

\section{Fatigue and Nutritional status}

Fatigue was assessed using the Functional Assessment of Cancer Therapy-Fatigue Subscale (FACT-F) [16]. Nutritional status was

*Corresponding author: Wolfgang Marx, Centre for Dietetics Research, School of Human Movement Studies, University of Queensland, Brisbane, Queensland, Australia, Tel: +61 418398 446; E-mail:wolfgang.marx@student.bond.edu.au

Received November 27, 2013; Accepted November 27, 2013; Published January 24, 2014

Citation: Marx WM, Teleni L, Ferguson M, Walpole E, Isenring EA (2014) The Effect of Chemotherapy on Serum Carnitine Levels and Fatigue in Chemotherapy Naïve Medical Oncology Patients: A Pilot Study. Carnitine, Chemotherapy and Fatigue. J Nutr Disorders Ther 4: 131. doi:10.4172/2161-0509.1000131

Copyright: @ 2014 Marx WM, et al. This is an open-access article distributed unde the terms of the Creative Commons Attribution License, which permits unrestricted use, distribution, and reproduction in any medium, provided the original author and source are credited. 
Citation: Marx WM, Teleni L, Ferguson M, Walpole E, Isenring EA (2014) The Effect of Chemotherapy on Serum Carnitine Levels and Fatigue in Chemotherapy Naïve Medical Oncology Patients: A Pilot Study. Carnitine, Chemotherapy and Fatigue. J Nutr Disorders Ther 4: 131. doi:10.4172/2161-0509.1000131

Page 2 of 4

assessed by an Accredited Practicing Dietitian trained in the use of the validated Patient Generated Subjective Global Assessment (PG-SGA) [17]. Symptoms that adversely impacted on dietary intake for the 2 weeks prior to assessment were captured within the PG-SGA.

\section{Statistical analysis}

Baseline characteristics were compared between genders using independent t-test analysis for continuous variables and chi-square or Fisher's exact test for categorical variables. The mean change in continuous variables between two time-points was assessed using paired t-tests. Associations between continuous variables were tested with linear regression, paired t-test or Mann-Whitney test. Statistical significance was set at $\mathrm{p}<0.05$.

\section{Results}

\section{Screening, enrolment and withdrawals}

Figure 1 Documents the recruitment process, including the reason for exclusions and withdrawals. Of the 35 patients enrolled, 27 patients completed the study. There were no statistical differences in patient demographics for those who did or did not provide full data and the sample was representative of those meeting the study inclusion criteria.

\section{Patient demographics and baseline characteristics}

In Table 1, baseline socio-demographic and clinical characteristics of the study population were documented. Patients were predominantly male $(65 \%, 22 / 35)$ with a mean age of $52 \pm 16.2$ years, were mostly wellnourished $(74 \%, 26 / 35)$, had a BMI within the normal or overweight range $(91 \%, 32 / 35)$, and were weight stable $(89 \%, 28 / 31)$.

The primary diagnoses of the patients included in this study varied considerably. However, Haematological and lymphoma cancers made up $48.6 \%$ (17/35) of the primary diagnoses of the study sample. There was also considerable variation in the chemotherapy regimens prescribed to patients in our study; however, $77 \%$ (27/35) of patients

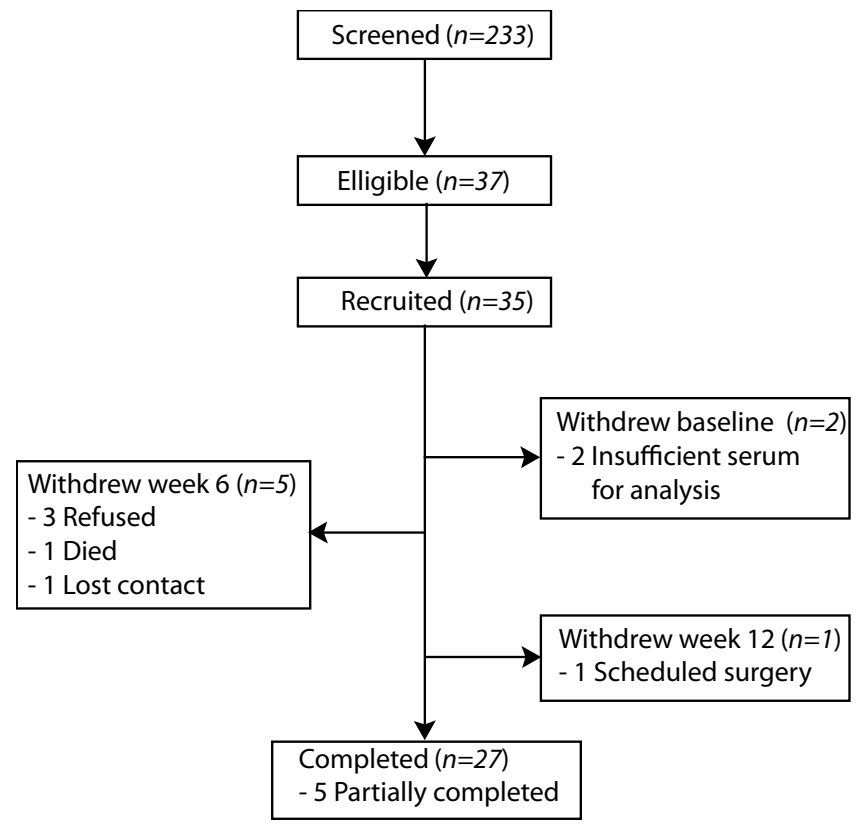

Figure 1: Participant flow diagram.

\begin{tabular}{|c|c|}
\hline Characteristic & Baseline \\
\hline Gender (M:F) & $22: 13$ \\
\hline Age (years) & $52 \pm 16.2$ \\
\hline \multicolumn{2}{|l|}{ Type of cancer } \\
\hline - $\quad$ Lung & 4 \\
\hline - Breast/Ovary & 4 \\
\hline - Colorectal & 4 \\
\hline - Haematological & 10 \\
\hline - Lymphoma & 7 \\
\hline - Other/Unknown & 6 \\
\hline Average number of chemotherapy cycles & $6 \pm 4$ \\
\hline \multicolumn{2}{|l|}{ BMI (kg/m2) } \\
\hline - $\quad$ Underweight & 3 \\
\hline - $\quad$ Normal weight & 11 \\
\hline - Overweight/Obese & 21 \\
\hline Significant weight loss past month (Yes/No) & $3 / 28$ \\
\hline \multicolumn{2}{|l|}{ Nutrition Impact symptoms } \\
\hline - $\quad$ Eating well (Yes/No) & $22 / 13$ \\
\hline - $\quad$ Appetite (Normal/Altered) & $27 / 8$ \\
\hline - $\quad$ Nausea (Yes/No) & $3 / 32$ \\
\hline - $\quad$ Vomiting (Yes/No) & $2 / 33$ \\
\hline - $\quad$ Taste (Normal/Altered) & $1 / 34$ \\
\hline - $\quad$ Early satiety(Yes/No) & $5 / 30$ \\
\hline - $\quad$ Other & $6 / 29$ \\
\hline SGA B/C (Malnourished) & 9 \\
\hline PG-SGA score (mean (range)) & $4(1-17)$ \\
\hline
\end{tabular}

Table 1: Baseline characteristics of 35 chemotherapy naïve patients.

underwent doxorubicin, ifosfamide or platinum based chemotherapy regimens.

\section{Chemotherapy-regimen, Carnitine and Fatigue}

At baseline, all patients had sufficient free carnitine levels with $10 \%(3 / 31)$ acyl: free carnitine ratio deficient. There was no significant increase in the prevalence of carnitine deficiency (total, free or acyl: free ratio) during the study. In fact, the three patients that possessed a deficient acyl: free carnitine ratio at baseline was no longer deficient by week 12. Additionally, no relationship between any measure of carnitine and fatigue was found at any time point.

While the majority of patients in this sample were undergoing regimens that have been implicated in the disruption of carnitine metabolism as well as cancer-related fatigue, our analysis showed no association between these regimens and any measure of carnitine deficiency or fatigue.

\section{Discussion}

Carnitine deficiency was not found to be a concern in a small consecutive sample of medical oncology patients undergoing chemotherapy. Disrupted carnitine metabolism may result in chemotherapy-related fatigue. Previous clinical trials have examined the effects of carnitine supplementation in resolving carnitine deficiencies and its association with fatigue in this population [14,1823]. The majority of these trials have found carnitine supplementation to significantly improve both carnitine status and measures of fatigue with minimal adverse effects. However, due to the findings of recent of a potential link between dietary carnitine and an increase in cardiovascular disease, the safety of carnitine supplementation needs to be further investigated [24]. 
Citation: Marx WM, Teleni L, Ferguson M, Walpole E, Isenring EA (2014) The Effect of Chemotherapy on Serum Carnitine Levels and Fatigue in Chemotherapy Naïve Medical Oncology Patients: A Pilot Study. Carnitine, Chemotherapy and Fatigue. J Nutr Disorders Ther 4: 131. doi:10.4172/2161-0509.1000131

Page 3 of 4

While large scale studies on the prevalence of carnitine deficiency are lacking in this population, previous trials have found a significant proportion of patients were carnitine deficient [23]. In contrast, our study was unable to find consistent signs of carnitine deficiencies in any of the participants and consequently, no correlation between carnitine levels and fatigue was found. In fact, free carnitine levels were on average, $9 \mu \mathrm{mol} / \mathrm{L}$ higher than healthy participants from previous research [10]. However, while non-significant, there was a downward trend in the mean of both free and total carnitine status during this study which could indicate that a longer study duration may have resulted in a significant decline in carnitine levels (Table 2).

There are many factors that affect carnitine metabolism and may explain the associations found within this study as well as the lack of carnitine deficiencies. Previous research has shown that while serum carnitine levels remained stable throughout treatment, urinary excretion was significantly elevated $[8,25]$. Considering that most participants were well nourished, and adequate dietary carnitine intake throughout chemotherapy treatment appeared sufficient to prevent deficiency. Additionally, as skeletal muscle is the major storage site for carnitine, muscle biopsy represents the most accurate method of carnitine analysis; however, this was considered too invasive for the studied population.

Lastly, the relatively small sample size ( $\mathrm{n}=35)$ employed may have been unable to detect significant changes in carnitine status and future trials should aim to recruit greater numbers in order to increase the statistical power. However, while the number of participants included in this trial was small, it was similar to multiple previous trials in this area with some studies recruiting between 5-40 participants. For example, Rogalidou et al. [6] investigated the effect of chemotherapy on serum carnitine levels in 40 participants using a similar study design; however, this study recruited children and adolescents only and may not be representative of carnitine metabolism in an adult population. Despite the limitations of our study, this pilot study provides a greater insight into the carnitine status of adult chemotherapy patients in a real clinical setting. By measuring carnitine status over three extended time points, this provides a greater understanding into the carnitine status of chemotherapy patients over their full treatment as opposed to acute changes only.

\section{Conclusion}

Carnitine deficiency was detected at baseline in a small portion of the study population; however, this did not persist to subsequent time points. Consequently, a relationship between carnitine status and fatigue was not demonstrated. Several possible explanations for these results have been discussed which highlight the need for further studies in this area. In particular, patient nutrition status, prescribed chemotherapy regimen, and chemotherapy cycle have all been identified as potentially affecting carnitine status and should be addressed in future studies that examine a larger population.

\begin{tabular}{|c|c|c|c|}
\hline Time point & Baseline & $\mathbf{6}$ weeks & $\mathbf{1 2}$ weeks \\
\hline Fatigue $($ Fact-F) & $114 \pm 30.7$ & $112 \pm 30.7$ & $112 \pm 31.7$ \\
\hline Total carnitine $(\mu \mathrm{mol} / \mathrm{L} \pm \mathrm{SD})$ & $75.2 \pm 17.5$ & $70.95 \pm 18$ & $69.04 \pm 13.7$ \\
\hline Free carnitine $(\mu \mathrm{mol} / \mathrm{L} \pm \mathrm{SD})$ & $59.8 \pm 15.6$ & $56.4 \pm 16$ & $54.6 \pm 10.4$ \\
\hline Acyl-carnitine $(\mu \mathrm{mol} / \mathrm{L} \pm \mathrm{SD})$ & $15.42 \pm 3.89$ & $14.52 \pm 5.09$ & $13.41 \pm 5.37$ \\
\hline Acyl-carnitine ratio & $0.27 \pm 0.07$ & $0.27 \pm 0.1$ & $0.26 \pm 0.05$ \\
\hline
\end{tabular}

Table 2: Fatigue and mean carnitine levels at each time point.

\section{References}

1. Stasi R, Abriani L, Beccaglia P, Terzoli E, Amadori S (2003) Cancer-related fatigue: evolving concepts in evaluation and treatment. Cancer 98: 1786-1801.

2. Seline KG, Johein $H(2007)$ the determination of I-carnitine in several food samples. Food Chemistry 105: 793-804.

3. Steiber A, Kerner J, Hoppel CL (2004) Carnitine: a nutritional, biosynthetic, and functional perspective. Mol Aspects Med 25: 455-473.

4. Khositseth A, Jirasakpisarn S, Pakakasama S, Choubtuym L, Wattanasirichaigoon D (2011) Carnitine levels and cardiac functions in children with solid malignancies receiving doxorubicin therapy. Indian J Med Paediatr Oncol 32: 38-42.

5. Haschke M, Vitins T, Lüde S, Todesco L, Novakova K, et al. (2010) Urinary excretion of carnitine as a marker of proximal tubular damage associated with platin-based antineoplastic drugs. Nephrol Dial Transplant 25: 426-433.

6. Rogalidou M, Evangeliou A, Stiakaki E, Giahnakis E, Kalmanti M (2010) Serum carnitine levels in childhood leukemia. J Pediatr Hematol Oncol 32: e61-69.

7. Hockenberry MJ, Hooke MC, Gregurich M, McCarthy K (2009) Carnitine plasma levels and fatigue in children/adolescents receiving cisplatin, ifosfamide, or doxorubicin. J Pediatr Hematol Oncol 31: 664-669.

8. Mancinelli A, D'Iddio S, Bisonni R, Graziano F, Lippe P, et al. (2007) Urinary excretion of L-carnitine and its short-chain acetyl-L-carnitine in patients undergoing carboplatin treatment. Cancer Chemother Pharmacol 60: 19-26.

9. Marthaler NP, Visarius T, Küpfer A, Lauterburg BH (1999) Increased urinary losses of carnitine during ifosfamide chemotherapy. Cancer Chemother Pharmacol 44: 170-172.

10. Vinci E, Rampello E, Zanoli L, Oreste G, Pistone G, et al. (2005) Serum carnitine levels in patients with tumoral cachexia. Eur J Intern Med 16: 419-423.

11. Yaris N, Akyüz C, Coşkun T, Büyükpamukçu M (2002) Serum carnitine levels of pediatric cancer patients. Pediatr Hematol Oncol 19: 1-8.

12. Peluso G, Nicolai R, Reda E, Benatti P, Barbarisi A, et al. (2000) Cancer and anticancer therapy-induced modifications on metabolism mediated by carnitine system. J Cell Physiol 182: 339-350.

13. Visarius TM, Bähler H, Küpfer A, Cerny T, Lauterburg BH (1998) Thiodiglycolic acid is excreted by humans receiving ifosfamide and inhibits mitochondrial function in rats. Drug Metab Dispos 26: 193-196.

14. Cruciani RA, Dvorkin E, Homel P, Malamud S, Culliney B, et al. (2006) Safety, tolerability and symptom outcomes associated with L-carnitine supplementation in patients with cancer, fatigue, and carnitine deficiency: a phase I/II study. J Pain Symptom Manage 32: 551-559.

15. Hardy DT, Preece MA, Green A (2001) Determination of plasma free carnitine by electrospray tandem mass spectrometry. Ann Clin Biochem 38: 665-670.

16. Yellen SB, Cella DF, Webster K, Blendowski C, Kaplan E (1997) Measuring fatigue and other anemia-related symptoms with the Functional Assessment of Cancer Therapy (FACT) measurement system. J Pain Symptom Manage 13: $63-74$

17. Bauer J, Capra S, Ferguson M (2002) Use of the scored Patient-Generated Subjective Global Assessment (PG-SGA) as a nutrition assessment tool in patients with cancer. Eur J Clin Nutr 56: 779-785.

18. Cruciani RA, Dvorkin E, Homel P, Culliney B, Malamud S, et al. (2009) L-carnitine supplementation in patients with advanced cancer and carnitine deficiency: a double-blind, placebo-controlled study. J Pain Symptom Manage 37: 622-631.

19. Gramignano G, Lusso MR, Madeddu C, Massa E, Serpe R, et al. (2006) Efficacy of I-carnitine administration on fatigue, nutritional status, oxidative stress, and related quality of life in 12 advanced cancer patients undergoing anticancer therapy. Nutrition 22: 136-145.

20. Graziano F, Bisonni R, Catalano V, Silva R, Rovidati S, et al. (2002) Potential role of levocarnitine supplementation for the treatment of chemotherapyinduced fatigue in non-anaemic cancer patients. $\mathrm{Br} \mathrm{J}$ Cancer 86: 1854-1857.

21. Macciò A, Madeddu C, Gramignano G, Mulas C, Floris C, et al. (2012) A randomized phase III clinical trial of a combined treatment for cachexia in patients with gynecological cancers: evaluating the impact on metabolic and inflammatory profiles and quality of life. Gynecol Oncol 124: 417-425. 
Citation: Marx WM, Teleni L, Ferguson M, Walpole E, Isenring EA (2014) The Effect of Chemotherapy on Serum Carnitine Levels and Fatigue in Chemotherapy Naïve Medical Oncology Patients: A Pilot Study. Carnitine, Chemotherapy and Fatigue. J Nutr Disorders Ther 4: 131. doi:10.4172/2161-0509.1000131

22. Mantovani G, Maccio A, Madeddu C, Serpe R, Massa E, et al. (2010) Randomized phase III clinical trial of five different arms of treatment in 332 patients with cancer cachexia. Oncologist 15: 200-211.

23. Cruciani RA, Zhang JJ, Manola J, Cella D, Ansari B, et al. (2012) L-carnitine supplementation for the management of fatigue in patients with cancer: an eastern cooperative oncology group phase III, randomized, double-blind, placebo-controlled trial. J Clin Oncol 30: 3864-3869.
24. Koeth RA, Wang Z, Levison BS, Buffa JA, Org E, et al. (2013) Intestinal microbiota metabolism of L-carnitine, a nutrient in red meat, promotes atherosclerosis. Nat Med 19: 576-585.

25. Heuberger W, Berardi S, Jacky E, Pey P, Krähenbühl S (1998) Increased urinary excretion of carnitine in patients treated with cisplatin. Eur $\mathrm{J}$ Clin Pharmacol 54: 503-508. 